\title{
Design and Analysis of Smart Sensing System for Animal Emotions Recognition
}

\author{
Erick Alphonce \\ Massawe \\ Nelson Mandela African \\ Institution of Science and \\ Technology, \\ Arusha, Tanzania
}

\author{
Michael Kisangiri \\ Nelson Mandela African \\ Institution of Science and \\ Technology, \\ Arusha, Tanzania
}

\author{
Shubi Kaijage \\ Nelson Mandela African \\ Institution of Science and \\ Technology, \\ Arusha, Tanzania
}

\author{
Padmanabhan \\ Seshaiyer \\ Department of \\ Mathematical Sciences \\ College of Science \\ George Mason University \\ Virginia, United states of \\ America
}

\begin{abstract}
Recently, animal emotion recognition has become an important field for developing intelligent systems for tracking and monitoring rhinos and elephants. In this work, a smart sensing system that helps in detecting animal emotions based on information from physiological parameters obtained from sensors attached on animal body, has been designed. The signals are continuously obtained from a heart rate sensor,galvanic skin resistance sensorand body temperature sensor. After amplifying and filtering of the signals from the sensors are done, they are processed in the microcontroller and transmitted wirelessly using GSM modem and ZigBee technologies. The signals which are received from the system are displayed and stored in the database where they are analyzed visually for patterns. The four basic emotions parameters observed in this project are happy (excited), sad, angry and neutral (relaxed). In this research dog have been used for the pilot study.
\end{abstract}

\section{General Terms}

Wireless sensor network, circuit simulation, animal emotion, circuit designing

\section{Keywords}

Animal emotion, smart sensors, heart rate, skin temperature, skin conductance, voice control, zigbee, K-means clustering algorithm, animal, rhino, elephant

\section{INTRODUCTION}

Research on emotion detection is a very challenging field that target methods to make effective intelligent sensor network for endangered animal's species that can report poaching incidence before happening. Recent researches have been focused on tracking the animal's positions only by using GPS attached on the animal body.

Emotions play critical role in rational and intelligent behaviors. It is a mental state that does not arise through free will and is often accompanied by physiological changes. These changes need to be monitored as they contain information about different types of emotions which will assist in understanding behaviors.

As observational methods in animal behavior research have always gone hand in hand with the development of new technologies we started to customize available sensor node technology and apply it to collect and propagate data from living animals. Currently, we are equipping cows and dogs with standard sensor nodes as a pilot study and developing a custom sensor suite, adapted to their specific requirements. Each sensor is contained in a module (referred to as sensor board) that performs signal conditioning and basic feature extraction. Sensor nodes are attached to a cow and dog, using a leather harness, fitted with a pocket for the sensing equipment. The long term goal is to attach, or even implant, the sensor nodes to rhinos and elephants.

The aim of this paper is to design a invasive system which will be capable of recognizing animal emotions based on measuring physiological parameters of the animal body using smart sensors.

In this paper we will design a sensor based system which is either in direct contact with the animal body (invasive) or indirectly (non-invasive). These sensors based system will also have a positive impact animal on health monitoring system.

\section{RELATED WORKS}

In the past different approaches and methods have been used to detect and evaluate human emotions. The most common ones include the use of electroencephalogram (EEG) signals, facial expressions, speech, body gestures, textual information and physiological signals.

EEG signals have been used to analyse the nervous system to provide information about different types of emotions. Cameras and image processing techniques are used to detect changes in facial expressions using external stimulus to excite specific emotions. Voice recognition systems monitor the tone of speech and hence providing information about an individual's emotion.Textual emotion recognition is based on words or sentences used in a developed chatting system.Emotion recognition using body movement and gesture evaluates emotion based on specific actions or dances assigned and naturally occurring.

In this paper, an emotion recognition system is developed using physiological signals. These signals are obtained from a heart rate sensor, a skin temperature sensor and a skin conductance sensor and stored for data analysis and feature extraction. The four basic emotions considered in this paper are happy (excited), sad, angry and neutral (relaxed).

A number of relevant wildlife tracking projects alreadyexist. Of these, there are two of particular interest for our project.The Global Positioning System(GPS) tracking collars allow for remote detection of the collared animal's position.The GPS is used to record the animal's exact location and store the readings at pre-set intervals. Locations are logged and can then be downloaded in various ways. The older GPS collars are used to store the data until the collar was retrieved. Now, most GPS collars allow the data to be downloaded remotely. GPS/GSM collars use GSM cell phone signal to download data. Store-onboard GPS collars can store data which is then downloaded 
remotely using a handheld Ultra high frequency (UHF) device. SAT/GPS collars use global Satellites to transmit the position and other data to a user's server/desktop. The very high frequency (VHF)tracking collar stands also known as a pulse collar[1]. VHF transmitters attached to a study animal emit a pulsed radio signal allowing a person to physically locate and observe the animal by homing into the signal using a receiver and directional antenna. The two tracking projects works in a communication scenario similar to ours.Our project has combined the two tracking techniquescoming up with a hybrid network which is more efficient with less power consumption[2]

\subsection{Cattle and Dog behaviors}

Cows are known to display subtle behaviors to express their emotions. While these expressions are hard to see, one can carefully observe patterns in their behavior. For instance the amount of white showing in cow's eyes are related to stress and frustration or even pain. Also, it has been observed that calves that are separated from their mothers approached new objects with more reluctance than other calves [11]. A Cambridge University study found that young heifers are excited upon discovering they can accomplish something physically such as opening a gate themselves by pressing a button with their noses, but not if the gate opens randomly. Besides intellectual or physical stimuli researchers have also observed that grazing cows often opt to stand near others with whom they are most familiar.However, many dairy farmers choose to separate cows according to productivity and reproductive state. The study suggests that herders may benefit more from allowing cow cliques to remain together [3].

Similar behaviours have been observed in dogs as well. For example some dogs are known to show show separation-related behaviourthat may have underlying negative emotional states.Other studies have suggested that dogs can experience negative emotions in a similar manner to people, including the equivalent of certain chronic and acute psychological conditions such as depression. Some researchers also have observed dogs showing jealousy against other dogs that maybe rewarded more.Researchers also believe that dogs show empathy which is feeling what others feel. Empathy is the ability to not only detect what others feel but also to experience that emotion yourself [4].

Dr. Peter Pongracz from EotvosLorand University, Budapest, and colleagues have produced evidence that dog barks contain information that people can understand. Their research suggests that even people who have never owned a dog can recognize the emotional 'meaning' of barks produced in various situations, such as when playing, left alone and confronted by a stranger[5].

\section{SYSTEM DESIGN}

The system consists of transmitter section and receiver section. The transmitter section consists of physiological sensors, signal conditioning circuit, an Arduino Mega 2560 R3 and Zigbee module. Receiver section consists of zigbee coordinator and computer for data storage. The data from the system is wirelessly transmitted to a computer where it is displayed and stored.

We use commercial, off-the-shelf Sensors, basic processing (8bit, $7 \mathrm{MHz})$ and radio communication $(900 \mathrm{MHz})$. Sensors are attached to the cattle/Dog using a leather harness, onto which a bag (containing the sensor) is fastened with Velcro. The designed leather harness consists of a leather band, wrapped around the rib cage, and a stiff wire loop around the neck to keep it from sagging off to the side. In order to reduce processing and memory complexity we have developed "smart" sensing modules to perform - in hardware - as much of the signal conditioning and feature extraction as possible. At present, we have two functioning sensor boards: one for heart rate analysis and one for body temperature analysis.

\subsection{Heart Rate Sensor}

The most common heart rate variability at rest can be due to serious heart problems, respiratory problems and emotional imbalance i.e. stress, panic attacks, anxiety and depression. In this work our interest is in finding relationship between heart rate and emotions.

PSL-iECG2 is a small 2-channel ECG module that outputs ECG waveforms and heartbeats. DC $5 \mathrm{~V}$ input power can be used to power Arduino board without any other power supply which is very convenient. Amplification is a $750 \mathrm{~V} / \mathrm{V}$. The notch filter can be adjusted to $50 \mathrm{~Hz}$ or $60 \mathrm{~Hz}$ by the notch filter selectable switch. The module outputs a high-quality ECG signal by applying the low noise and optimal filter design.

PSL-iECG2 guarantees electrical safety through isolation power and signal during measuring. For the high quality signal input the premium lead cable with shield is provided, and the cable adopts a stereo jack to connect simply[6]

In order to process the ECG features we first subtract the global mean value of the raw signal that might be shifted up or downwards by the recording device. The signal is filtered with a low pass filter with $f c=90 \mathrm{~Hz}$ to remove high frequency noise, a very sharp high pass $f_{c}=0.5 \mathrm{~Hz}$ to eliminate low frequency drifts that occur when the subject is moving and then a notch filter at $f n=50 \mathrm{~Hz}$ that removes influences of the power line. We detected the ECG graphical deflections complexes (QRS) by the following Algorithm [7]. This is a derivative based method for detecting QRS complexes of the ECG signal consisting of a weighted sum of the smoothed first derivative and the approximated second derivative[8].

$$
\begin{gathered}
y(n)=(a \times x(n)-x(n-2))+(b \times x(n)-x(n)-x(n-2) \\
+x(n-4)
\end{gathered}
$$

where $\mathrm{x}(\mathrm{n})$ is the input signal, $a$ and $b$ are the weight parameters for the balance between the smoothed three point first derivative and the approximated second derivative and $y(n)$ is the output of this stage. $a$ and $b$ are set to 1.3 and 1.1 according to [7]. A further smoothing of the result is obtained by the introduction of an M-point moving average filter [9]

The resulting signal is scanned with an adaptive threshold that is defined by (3)

$y_{f i l t}=\frac{1}{M} \sum_{j=0}^{M-1} y(n-j)$

$\operatorname{thres}(n)=\frac{\max [n-k, n+k]-\min [n-k, n+K]}{\alpha}$

The result is a list of samples each indicating the onset of a single QRS complex. This list is used to calculate a set of different features like heart rate (HR), heart rate variability (HRV) and the inter beat interval (IBI) between consecutive heart beats. The HRV for example is influenced by the sympathetic and parasympathetic vagus nerve and therefore a good indicator for the temporary dominance of one of them.

The HR is calculated simply by using the difference between two consecutive detected QRS complexes $\left(t_{H B}\right)$

$$
\mathrm{HR}(\mathrm{n})=\frac{1}{60} \cdot \frac{\mathrm{t}_{\mathrm{HB}(\mathrm{n})-\mathrm{t}_{\mathrm{HB}(\mathrm{n}-1)}}}{\mathrm{fs}}
$$

However, in order to make it more stable against artifacts and 
irregularities of the heart function we introduce a logic that calculates the mean duration of the previous IBIs and checks if the next heart beat occurs within a range of $+/-15 \%$. If this constraint is violated, then we assume an out of range increase of the heart rate and correct the signal by introducing a beat at the expected position. This prevents missed QRS complexes or anomalies from influencing the HR. The HR itself is used as a feature as well as the previously mentioned common features derived from it[8].

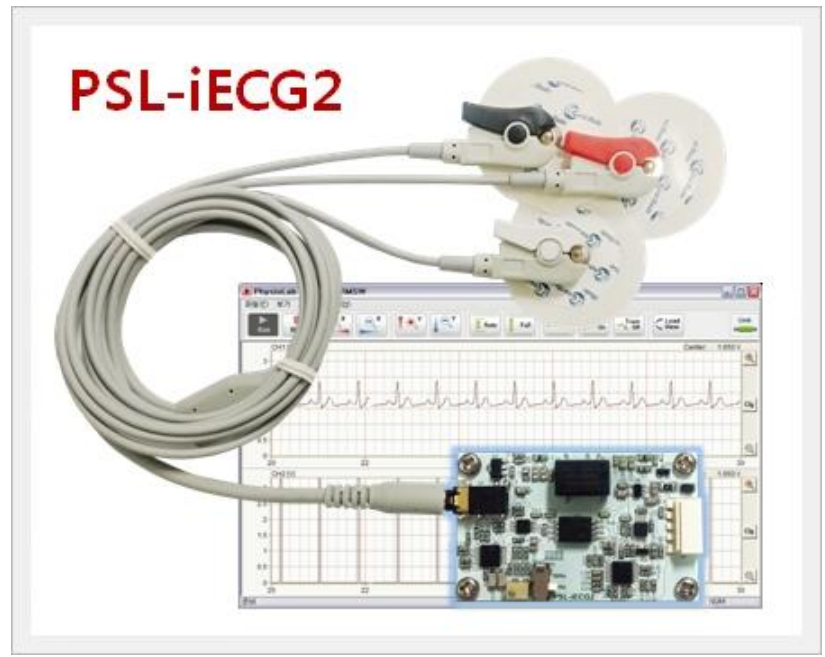

Fig 1. PSL-iECG2 (Mini-size 2ch ECG sensor module with isolation)

\subsection{Body Temperature Sensor}

In this research LM35 is used as a temperature sensor. The LM35 series are precision integrated-circuit temperature sensors with an output voltage that is linearly proportional to the Centigrade temperature. Thus the LM35 has an advantage over the linear temperature sensors that are calibrated in Kelvin, as the user is not required to subtract a large constant voltage from the output to obtain a convenient Centigrade scaling. LM35 does not require any external calibration or trimming to provide the typical accuracies of $\pm 1 / 4^{\circ} \mathrm{C}$ at the room temperature and $\pm 3 / 4^{\circ} \mathrm{C}$ over a full $-55^{\circ} \mathrm{C}$ to $+150^{\circ} \mathrm{C}$ temperature range. The low output impedance, linear output, and precise inherent calibration of the LM35 sensor make interfacing to readout or control circuitry especially easy[10].

The device is used with single power supplies, or with plus and minus supplies. As the LM35 sensor draws only $60 \mu \mathrm{A}$ from the supply, it has very low self-heating of less than $0.1^{\circ} \mathrm{C}$ in the still air. The LM35 is rated to operate over a $-55^{\circ} \mathrm{C}$ to $+150^{\circ} \mathrm{C}$ temperature range, while the $\mathrm{LM} 35 \mathrm{C}$ is rated for a $-40^{\circ} \mathrm{C}$ to $+110^{\circ} \mathrm{C}$ range $\left(-10^{\circ}\right.$ with improved accuracy).

There are a number of devices available for monitoring or observing body temperature. In this research, the aim was to go for a low-cost, compact, reliable, and accurate temperature sensor that is capable of monitoring the body temperature with ease and comfort.

As stated earlier, the output from the temperature sensor is an analog voltage. This output signal from the sensor is used as the input for the microcontroller through the analog port pin. The microcontroller is programmed to perform the required processing and conversion from a voltage value into a temperature value. The relationship between the voltage value and the temperature value is calculated by the following equation:

$$
\begin{gathered}
T\left({ }^{\circ} \mathrm{C}\right)=\frac{V_{\text {out }}-\mathrm{V}_{\text {oS }}}{\Delta \mathrm{V} / \Delta \mathrm{T}} \\
\text { Where } \\
\text { Vos }=\text { Ds offset, } 509 \mathrm{mV} \\
\Delta \mathrm{V} / \Delta \mathrm{T}=\text { Typical output gain, }+\frac{6.45 \mathrm{mV}}{{ }^{\circ} \mathrm{C}}
\end{gathered}
$$

The popular of all the electrical systems today employ some sort of microcontroller technology. A microcontroller's inexpensive, flexible, and self-sufficient design permits it to command almost any modern task that employs some form of embedded systems. From cars to refrigerators to handheld devices, microcontrollers play a dominant role in the development of many different products for many different companies.

\subsection{Microcontroller Overview}

In this research, the Microcontroller used is PIC XMEGA256A3.The PIC XMEGA256A3 includes a 16bit CPU, 16-bit timer,16-bit Sigma Delta Analog-to-Digital converter, brownout detector, Watchdog timer, USI module supporting SPI and I2C serial communication standards, and five low power modes drawing as little as $0.1 \mu \mathrm{A}$ standby current.

\subsection{Communication}

The system is designed to communicate with the computer wirelessly as well as using an RS232-USB. The wireless communication is achieved by using Zigbee technology. Zigbee is suitable for low rate data and secure networking. It has been widely used for monitoring applications.

It has a range of up to $40 \mathrm{~m}$. In this project we used XBee Series 2 OEM RF modules which operate within the ZigBee protocol and uses $2.4 \mathrm{GHz}$ frequency band. It requires a supply voltage of $3 \mathrm{~V}$ which is provided by the microcontroller and consumes power as low as $296 \mathrm{~mA}$

\section{CIRCUIT SIMULATION RESULTS}

The simulation of the circuit was done on Proteus Professional v 8.0 software package. The software of the system is written in the embedded $\mathrm{C}$ language. Figure 2 shows the simulation schematic of the system. The simulated system consists of the following components; microcontroller, body temperature sensor, heart rate monitor sensor, pulse generator, GSP, horns synchronization sensor and virtual terminals.

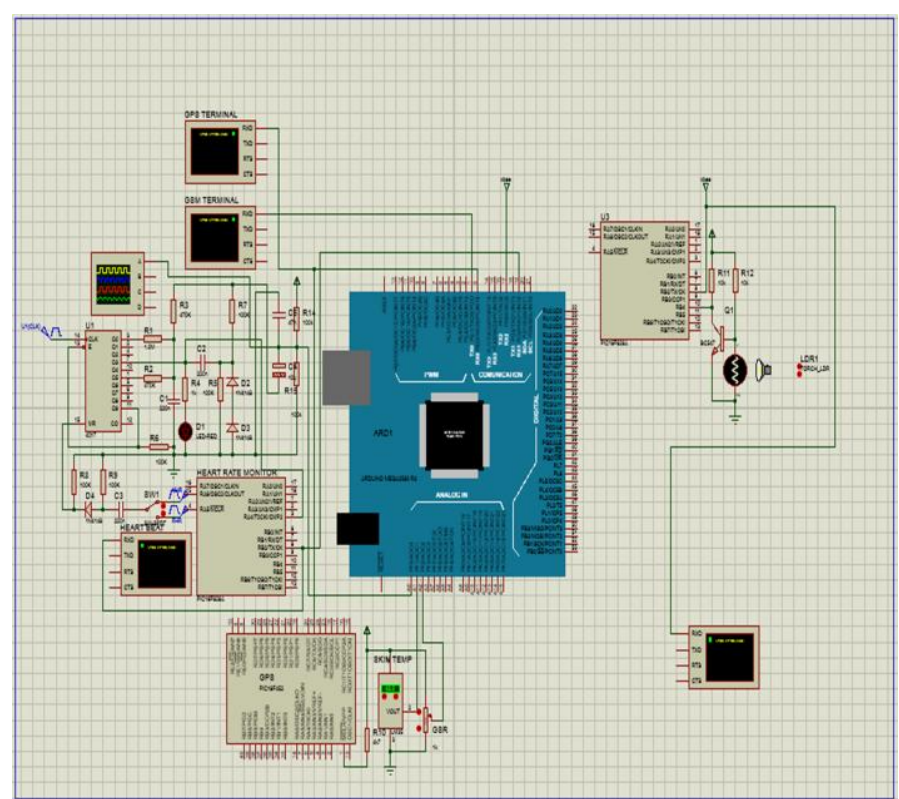

Figure 2. PROTEUS Circuit Simulation Schematic 


\section{CIRCUIT SIMULATION OUTPUT}

The circuit simulation output is as shown on the figure below. This is the output notifications messages from the system. The message displays the heart beat rates, body temperature and status of the horns syschronizations.It also shows response signal sent to drone,mobile phone and data base.

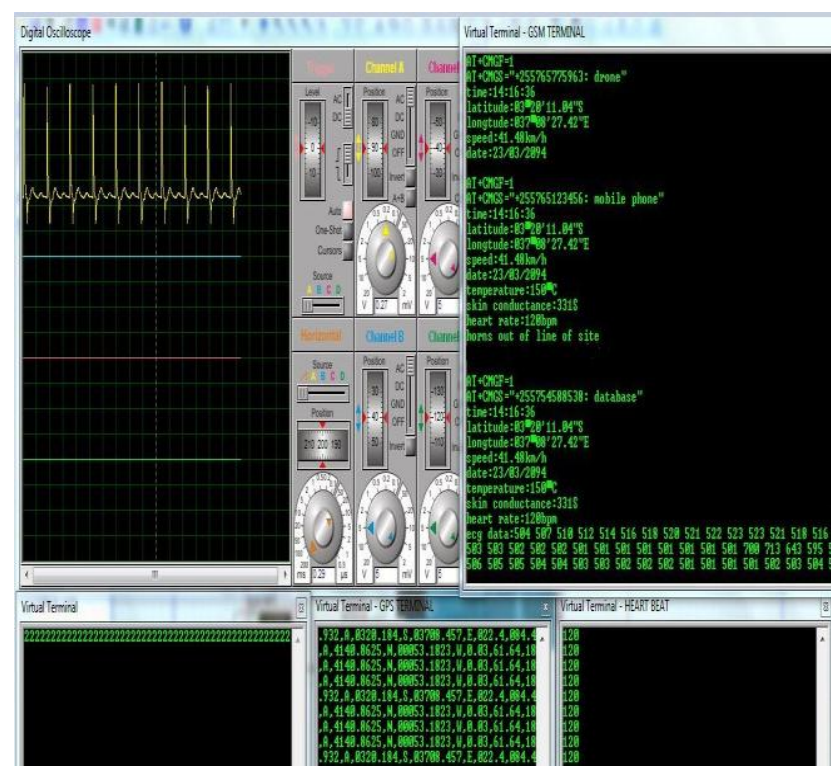

Figure 2. Circuit simulation output

\section{HARDWARE MODEL}

The developed system is in a shape of a belt with a surface area of 80inch $\mathrm{x}$ 3inch which is large enough to fit on cow and dog neck. The microcontroller and signal condition circuits reside on the top of the collar while the ECG sensor and body temperature sensor electrodes are mounted inside the surface in order to be in direct and easy contact with the animal body. The system is designed to monitor data from the animal body which is shown below in figure 4.

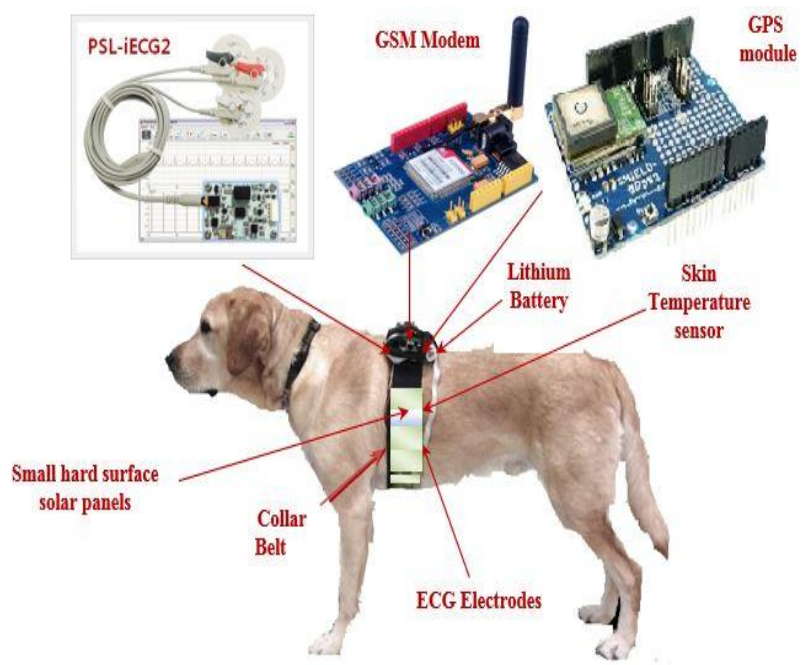

Figure 3. 3D model of the surface of the system with the sensors and electrodes.

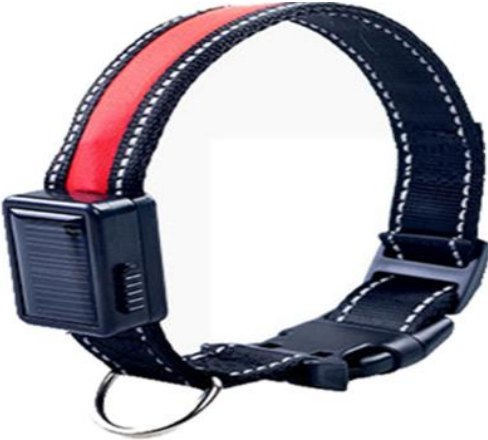

Figure 4. 3D model of the solar powered collar belt

The signal from the sensors are processed by PIC XMEGA256A3 microcontroller and sent wirelessly by the Zigbee router which is received at the other end by the Zigbee coordinator.

The coordinator is connected to the computer via the USB port. The data after being received is displayed using LabView and is stored in the database. The next step was to collect data from different dogs and observe the changes. This is explained in the results section below.

\section{RESULTS AND DISCUSSION}

Once the reliability of the sensors was tested, we started collecting data from dogs and cows as pilot studies for our project. This data was stored in order to help in finding obvious visual patterns and also use it for feature extraction. The results of heart beat, body temperature data are collected from subjects in happy (excited), sad, relaxed (neutral), and angry states have to be done.

The results proved that different emotions have an effect on the physiological signals which can be visualized by continuous monitoring. The heart rate variability were the dominant signals in this case. The heart rate values showed that, the higher the relaxed state, the lower the heart rate values get and vice versa, which proves that the heart rate values are directly related to the level of arousal.

\section{CONCLUSION AND FUTURE WORK}

In this paper, a real time emotion recognition system based on data provided by physiological sensors was developed. Physiological sensors were found to be the best approach to recognize emotional changes, as they provided information about changes that take place physiologically and are out of the animal's control. The sensors have been integrated and placed on the inner surface of the collar belt, for collecting data from the animal body. This design enabled an easy and comfortable physiological data monitoring system that can be used on antpoaching monitoring.

For future improvement and development of the system, the use of additional physiological sensors such as Voice control sensor, pressure sensor and respiration sensors is required. In order to get more information from the data other clustering methods should be looked into which can help in improving emotion recognition rate. The system once fully developed will be capable of extracting basic emotions i.e. happy, sad, angry and neutral from the physiological signals. It can be integrated with the drone which will help on immediately response to attend poaching incidence.

\section{REFERENCES}

[1] W. act. (2014). GPS and VHF tracking collars used for wildlife monitoring.

Available: 
http://wildlifeact.com/blog/gps-and-vhf-tracking-collarsused-for-wildlife-monitoring/

[2] Volunteerforever. (2014). VHF tracking collars. Available: https://www.volunteerforever.com/blogs_post/vhf-trackingcollars

[3] J. Balcombe. (2015). Learn how cows express their emotions.

Available: http://www.humanesociety.org/news/magazines/2015/1112/how-cows-expressemotions.html?referrer=https://www.google.com/

[4] U. o. Bristol. (2010). Dogs may be pessimistic too. Available: http://www.bris.ac.uk/news/2010/7261.html

[5] D. M. reporter. (2008). Living with humans has taught dogs morals, say scientists. Available: http://www.dailymail.co.uk/sciencetech/article1047481/Living-humans-taught-dogs-morals-sayscientists.html
[6] PhysioLab. Biosignal Sensing and Monitoring [Online]. Available: http://physiolab.en.ec21.com/PSL-iECG2_Minisize_2ch_ECG_sensor--9063127_9816937.html

[7] R. M. Rangayyan and N. P. Reddy, "Biomedical signal analysis: a case-study approach," Annals of Biomedical Engineering, vol. 30, pp. 983-983, 2002.

[8] A. Haag, et al., "Emotion recognition using bio-sensors: First steps towards an automatic system," in Tutorial and research workshop on affective dialogue systems, 2004, pp. $36-48$.

[9] P. Lang, et al., "International affective picture system (IAPS): Instruction manual and affective ratings. University of Florida; Gainesville," FL: 2005. Technical Report A61999 .

[10] T. Murugan, et al., "Embedded based Industrial Temperature Monitoring Systems using GSM," International Journal of Computer Applications, vol. 58, 2012. 